$\xi_{p}$

\title{
Various Characteristics of a Few Topological Indices for $\gamma \_$Stable Graphs
}

\author{
S.Manimekalai ${ }^{1 *}$, U.Mary $^{2}$ \\ ${ }^{1}$ Department of Mathematics, Dr. N. G. P. Arts and Science College, Coimbatore, India \\ ${ }^{2}$ Department of Mathematics, Nirmala College for Women, Coimbatore, India \\ *Corresponding authorE-mail: manimekalai@drngpasc.ac.in
}

\begin{abstract}
In this manuscript we have given various characteristics of some topological indices for $\gamma$ stable graphs and computation of Wiener index using python program for Complex graph structures which frequently appear in all chemical Structure.
\end{abstract}

Keywords: bipartite index; Computation index; $\gamma$ stable graphs; topological index.

\section{Introduction}

Mixture of elements bonded jointly to form basic building of compounds are Molecules. An undirected graph symbolize the topological configuration of a substance mix, when the arrangement of vertices specify the element of the substance, the arrangement of edges specify the links among the elements and $\mathrm{F}$ is a corresponding function of edges and vertices. Hence this graph is of a substance mix is called molecular graph.[1-5]

Calculating Wiener index for composite graph structure is given by simple formula and python program. Concerned readers can check this in the web https://repl.it/repls/ShabbyFirmCheief.

Wiener manifestation nature of $\gamma$ stable graph [7]also declared in this paper.

\section{Definition:}

\subsection{Wiener Index [9]:}

The Wiener manifestation $\mathrm{W}(\mathrm{G})$ of a linked graph $\mathrm{G}$ with set of vertices $V(G)$ is described as

$\mathrm{W}(\mathrm{G})$ is equal to $\sum_{\mathrm{u}, \mathrm{v} \in \mathrm{V}(\mathrm{G})} \mathrm{d}_{\mathrm{G}}(\mathrm{u}, \mathrm{v})$

Where $\mathrm{d}_{\mathrm{G}}(\mathrm{u}, \mathrm{v})$ represents the shortest path among $\mathrm{u}$ and $\mathrm{v}$ in $\mathrm{G}$.

\subsection{Theorem:}

The Wiener index of the Cartesian product $\mathrm{K}_{4,4} \times \mathrm{P}_{\mathrm{r}}=\mathrm{G}$ is given by $W(G)=d W\left(K_{4,4}\right)+\left[2 W\left(K_{4,4}\right)+64\right](d-1)+\sum_{i=1}(n-i-1)\left[2 W\left(K_{4,4}\right)+64+i 64\right]$

Where $d$ is number of copies of bipartite graphs, $i$ ranges from 1 to till the summation reaches zero.

\subsection{Python Program for Wiener index calculation of Cartesian Product $\mathbf{K}_{4,4} \times P_{r}$}

\section{Connection $=144$}

Output $=0$

Print("Enter the number of elements :")

Count $=\operatorname{int}($ input ()$)$

Value $=0$

Output $=($ count $*$ common $)$

For $\mathrm{i}$ in range $(1$, count $)$ :

Value $=($ count $-\mathrm{i}) *$ connection

Connection=connection+increment

Output=ouput+value

Print (output)

\subsection{Table for Wiener Index of Cartesian Product $\mathbf{K}_{4,4} \times \mathbf{P}_{\mathbf{r}}$ :}

\begin{tabular}{|c|c|c|l|l|}
\hline S.No & $\begin{array}{c}\text { Number of } \\
\mathbf{K}_{\mathbf{4}, \mathbf{4}}\end{array}$ & Wiener Index & $\begin{array}{c}\text { Number } \\
\text { of } \mathbf{K}_{\mathbf{4}, \mathbf{4}}\end{array}$ & Wiener Index \\
\hline $\mathbf{1}$ & $\mathbf{n}=\mathbf{1}$ & $\mathbf{W}(\mathbf{G})=\mathbf{4 0}$ & $\mathbf{n}=\mathbf{6}$ & $\mathbf{W}(\mathbf{G})=\mathbf{3 6 8 0}$ \\
\hline 2 & $\mathbf{n}=\mathbf{2}$ & $\mathbf{W}(\mathbf{G})=\mathbf{2 2 4}$ & $\mathbf{n}=7$ & $\mathbf{W}(\mathbf{G})=5544$ \\
\hline 3 & $\mathbf{n}=\mathbf{3}$ & $\mathbf{W}(\mathbf{G})=616$ & $\mathbf{n}=\mathbf{8}$ & $\mathbf{W}(\mathbf{G})=7936$ \\
\hline 4 & $\mathbf{n}=\mathbf{4}$ & $\mathbf{W}(\mathbf{G})=1280$ & $\mathbf{n}=9$ & $\mathbf{W}(\mathbf{G})=10920$ \\
\hline 5 & $\mathbf{n}=\mathbf{5}$ & $\mathbf{W}(\mathbf{G})=\mathbf{2 2 8 0}$ & $\mathbf{n}=\mathbf{1 0}$ & $\mathbf{W}(\mathbf{G})=14560$ \\
\hline
\end{tabular}

\section{General Formula}

\subsection{Theorem:}

The Wiener index of the Cartesian product $\mathrm{K}_{\mathrm{m}, \mathrm{n}} \times \mathrm{P}_{\mathrm{r}}=\mathrm{G}$ is given by th

$W(G)=d W\left(K_{m, n}\right)+\left[2 W\left(K_{m, n}\right)+(m+n)^{2}\right](d-1)+\sum_{i=1}(n-i-1)\left[2 W\left(K_{m, n}\right)+(2 n)^{2}+i(2 n)^{2}\right]$

Where $\mathrm{W}\left(\mathrm{K}_{\mathrm{m}, \mathrm{n}}\right)=(\mathrm{m}+\mathrm{n})^{2}-(\mathrm{m}-\mathrm{n})-\mathrm{mn}$

Where $d$ is number of copies of bipartite graphs, iranges from 1 to till the summation reaches non negative value.

Proof: 


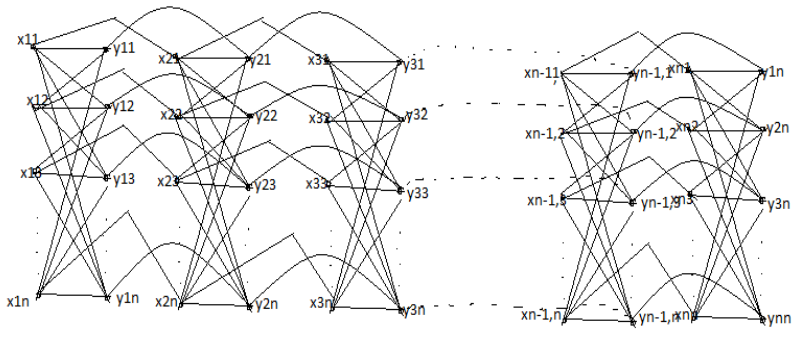

Cartesian Product Kn, $\mathrm{n}$ Pr

Fig.1: Cartesian Product $\mathrm{K}_{\mathrm{n}, \mathrm{n}} \times \mathrm{P}_{\mathrm{r}}$

The Wiener index of the Cartesian product $\mathrm{K}_{2,2} \times \mathrm{P}_{\mathrm{r}}=\mathrm{G}$ is given by $W(G)=d W\left(K_{2,2}\right)+\left[2 W\left(K_{2,2}\right)+16\right](d-1)+\sum_{i=1}(n-i-1)\left[2 W\left(K_{2,2}\right)+16+i 16\right]$

The Wiener index of the Cartesian product $\mathrm{K}_{3,3} \times \mathrm{P}_{\mathrm{r}}=\mathrm{G}$ is given by $W(G)=d W\left(K_{3,3}\right)+\left[2 W\left(K_{3,3}\right)+36\right](d-1)+\sum_{i=1}(n-i-1)\left[2 W\left(K_{3,3}\right)+36+i 36\right]$ The Wiener index of the Cartesian product $\mathrm{K}_{4,4} \times \mathrm{P}_{\mathrm{r}}=\mathrm{G}$ is given by $W(G)=d W\left(K_{4,4}\right)+\left[2 W\left(K_{4,4}\right)+64\right](d-1)+\sum_{i=1}(n-i-1)\left[2 W\left(K_{4,4}\right)+64+i 64\right]$ wh ere $d$ is number of copies of bipartite graphs, $i$ ranges from 1 to till the summation reaches zero.

The Wiener index of the Cartesian product $\mathrm{K}_{\mathrm{m}, \mathrm{n}} \times \mathrm{P}_{\mathrm{r}}=\mathrm{G}$ is given by $W(G)=d W\left(K_{m, n}\right)+\left[2 W\left(K_{m, n}\right)+(m+n)^{2}\right](d-1)+\sum(n-i-1)\left[2 W\left(K_{m, n}\right)+(2 n)^{2}+i(2 n)^{2}\right]$

\subsection{Python Program for Wiener calculation of Cartesian Product $\mathbf{K}_{\mathrm{m}, \mathrm{n}} \times \mathbf{P}_{\mathbf{r}}$}

Common $=8$

Vertex $=4$

Increment $=$ vertex $*$ vertex

Connection $=($ common $* 2)+$ increment

Output $=0$

Value $=0$

Print("Enter the number of elements :")

Count $=\operatorname{int}($ input ()$)$

Output $=($ count $*$ common $)$

For $\mathrm{i}$ in range $(1$, count):

Value $=($ count $-\mathrm{i}) *$ connection

Connection $=$ connection+increment

Output=ouput+value

Print (output)

\subsection{Result}

For two cycles $C_{1}$ and $C_{2}$ with any number of vertices $n$ connected by an single bridge the resulting graph $\mathrm{G}$, Wiener manifestation is $\mathrm{W}(\mathrm{G})=2 \mathrm{~W}\left(\mathrm{C}_{1}\right)+(\mathrm{n}+1)(\mathrm{n} / 2)$ or $\mathrm{W}(\mathrm{G})=\mathrm{W}\left(\mathrm{C}_{1}\right)+\mathrm{W}\left(\mathrm{C}_{2}\right)+(\mathrm{n}+1)(\mathrm{n} / 2)$

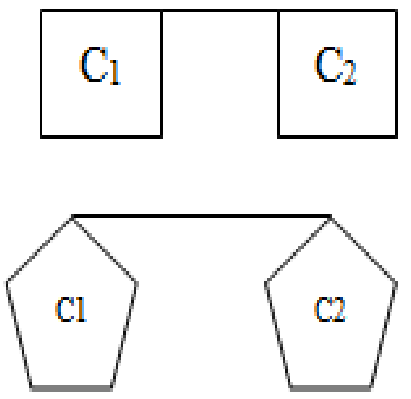

Fig.2: Graph G

\subsection{Result}

Consider any $(G)$ connected Graph with any number of vertices $n$, join any two non adjacent vertices by an single edge the resulting graph $G_{R}$, Total eccentricity [8]manifestation does not change. That is $\zeta(\mathrm{G})=\zeta\left(\mathrm{G}_{\mathrm{R}}\right)$

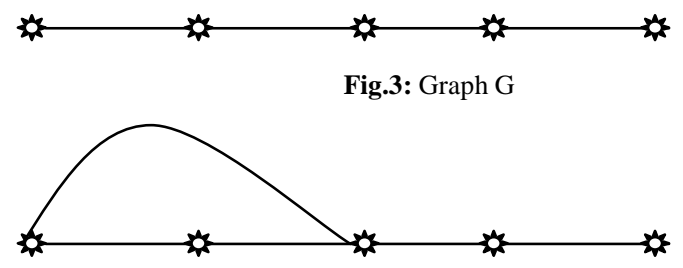

Fig.4: Graph $G_{R}$

\section{Definition}

\section{1 $\gamma$ - stable graph[12]}

$\gamma$ stable graph is nothing but if $\gamma\left(G_{x y}\right)=\gamma(G), \forall x$ and $y \in V(G)$, $\mathrm{x}$ and $\mathrm{y}$ are nonadjacent, $\mathrm{G}_{\mathrm{xy}}$ indicate the graph acquire by identifying $x$ and $y[12]$.

\subsection{The Zagreb Index [7,10]:}

For any simple, connected graph $\mathrm{G}$, the Zagreb manifestation of a graph $\mathrm{G}$ is clear by the following

$\mathrm{M}_{1}(\mathrm{G})=\sum_{\mathrm{v} \in \mathrm{V}(\mathrm{G})} \mathrm{d}_{\mathrm{G}}(\mathrm{v})^{2}$ or $\mathrm{M}_{1}(\mathrm{G})=\sum_{\mathrm{vu} \in \mathrm{E}(\mathrm{G})}\left[\mathrm{d}_{\mathrm{G}}(\mathrm{u})+\mathrm{d}_{\mathrm{G}}(\mathrm{v})\right]$

$M_{2}(G)=\sum_{u v} \in E(G) d_{G}(u) d_{G}(v)$

where $d_{G}(v)$ is the vertex degree in $G$.

\subsection{The F-Index Index [7,10]:}

$\mathrm{F}(\mathrm{G})=\sum_{\mathrm{v} \in \mathrm{V}(\mathrm{G})} \mathrm{d}_{\mathrm{G}}(\mathrm{v})^{3}$ or $\mathrm{F}(\mathrm{G})=\sum_{\mathrm{vu} \in \mathrm{E}(\mathrm{G})}\left[\mathrm{d}_{\mathrm{G}}(\mathrm{u})^{2}+\mathrm{d}_{\mathrm{G}}(\mathrm{v})^{2}\right]$

\subsection{Result}

Relation between Wiener, First Zagreb index, second Zagreb index and F-index of the following $\gamma$ stable graph $G$ and its complement Graph $\overline{\mathrm{G}}$ is given by

$\mathrm{W}(\mathrm{G})$ less than $\mathrm{M}_{1}(\mathrm{G})$

$\mathrm{M}_{1}(\mathrm{G})$ less than $\mathrm{M}_{2}(\mathrm{G})$

$\mathrm{M}_{2}(\mathrm{G})$ less than $\mathrm{F}(\mathrm{G})$ and

$\mathrm{W}(\overline{\mathrm{G}})$ less than $\mathrm{M}_{1}(\overline{\mathrm{G}})$

$\mathrm{M}_{1}(\overline{\mathrm{G}})$ less than $\mathrm{M}_{2}(\overline{\mathrm{G}})$

$\mathrm{M}_{2}(\overline{\mathrm{G}})$ less than $\mathrm{F}(\overline{\mathrm{G}})$

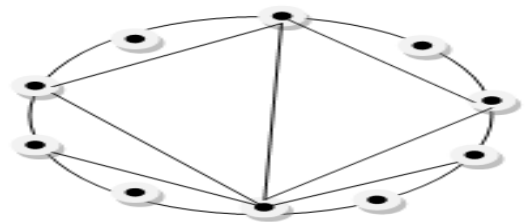

Fig.5: $\gamma$-Stable Graph G

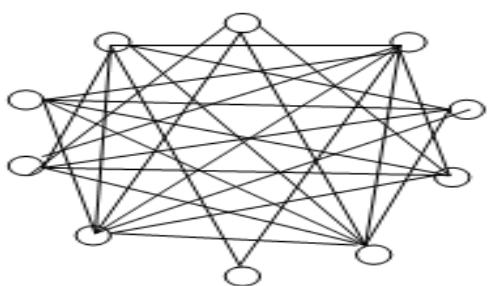

Fig.5: $\gamma$-Stable Graph $G^{c}$ 


\subsection{Result}

From the Figure 2 and $3, \gamma(G)$ and $\gamma\left(G^{c}\right)=2$, and for all possible $\mathrm{G}_{\mathrm{xy}}$,

(i) $\mathrm{W}\left(\mathrm{G}_{\mathrm{xy}}\right)<\mathrm{W}(\mathrm{G})$.

(ii) $\mathrm{M}_{1}\left(\mathrm{G}_{\mathrm{xy}}\right)>\mathrm{M}_{1}(\mathrm{G})$

(iii) $\mathrm{F}\left(\mathrm{G}_{\mathrm{xy}}\right)>\mathrm{F}(\mathrm{G})$

In common we get increased degree of vertex, when indentifying its end vertices. So in both (ii) and (iii) we get less index values for $\gamma$-stable graphs comparing with $\mathrm{G}_{\mathrm{xy}}$.

\section{Conclusion:}

The Wiener Topological index is established to the complex structure graph, that is Cartesian product of complete bipartite graph and path graph with two vertices, three vertices, four vertices and any number of vertices, and characteristics of topological indices for $\gamma$-stable graphs.

\section{References:}

[1] Diestel R, Graph Theory, Electronic Edition(2000) SpringerVerlag:

[2] Gonzalo Cerruela Garcia, Irene Luque Ruiz and Miguel Angel Gomez-Nieto, From Wiener Index to Molecules, J. Chem. Inf. Model. 2005, 45, 231-238

[3] Gross J, Yellen J,Graph Theory and Its Applications.(1991) CRC Press:

[4] Gutman I. A New Hyper-Wiener Index. Croat. Chem. Acta (2004), $77(1-2) 61-64$

[5] Ivanciuc O, Balaban AT,The Graph Description of Chemical Structures. In Topological Indices and Related Descriptors in QSAR and QSPR; Devillers, J., Balaban, A. T., Eds.; Gordon and Breach Science Publishers: The Netherlands, (1999) pp 59-167.

[6] Manimekalai S, Mary U, computation of zagreb index for complex graph structure.International journal of creative research thoughts (2018) 6 262-264

[7] Nilanjan De,Abu nayeem sk.Md,Computing the F index of nanostar dendrimers,Pacific Science Review A: Natural Science and Engineering,Volume 18,(2016)14-21

[8] Rouvray D, Balaban AT , Chemical Applications of Graph Theory. Applications of Graph Theory. Wilson, R. J., Beineke, L. W.,Eds.; Academic Press; (1979)pp 177-221.

[9] Sedlar J,On augmented eccentric connectivity index of graphs and trees, MATCH Commun. Math. Comput. Chem. 2012.68,325-342.

[10] Svante Janson, Philippe Chassaing, The center of mass of the ISE and the Wiener index of trees, Elect. Comm. in Probability. 9 (2004), 178-187

[11] Wei Gao,Muhammad Kamran Siddiqui, Muhammad Imran,Muhammad kamran Jamil,Mohammad reza Farahani (2016) Forgotten topological index of chemical structure in drugs,Saudi Pharmaceutical Journal.24,258-264

[12] Yamuna M and Karthika K, Excellent $\gamma$ - stable graphs, IOP Conf. Series: Materials Science and Engineering 263 (2017) 042116

[13] https://repl.it/repls/ShabbyFirmCheief. 